\title{
The Study of Inferior Alveolar Canal at the Lower Third Molar Apical Region With Cone Beam Computed Tomography
}

\author{
Trustiya Tudtiam ${ }^{\mathrm{a}}$, Rachatawan Leelarungsun ${ }^{\mathrm{a}}$, Lee Kian $\mathrm{Khoo}^{\mathrm{b}}$, Teeranut Chaiyasamut ${ }^{\mathrm{b}, \mathrm{e}}$, \\ Raweewan Arayasantiparb ${ }^{\mathrm{c}}$, Natthamet Wongsirichat ${ }^{\mathrm{b}, \mathrm{d}, \mathrm{e}}$
}

\begin{abstract}
Background: The aim of this study was to determine the distance between the impacted mandibular third molar (IMTM) and the inferior alveolar canal (IAC) at different reference points through panoramic radiographs and cone beam computed tomography (CBCT).
\end{abstract}

Methods: A total of 45 male and 45 female patients with bilateral IMTM at a mean age of 27 years were recruited for this study. With the aid of panaromic radiographs and $\mathrm{CBCT}$, the mandible width, the buccal bone width, IAC width, the lingual bone width, IAC height, the alveolar bone height, and the distance from the lowest part of the IMTM to the superior border of the canal were measured. Statistical analysis was done with independent samples $t$-test and Mann-Whitney $\mathrm{U}$ test for finding the difference between genders. Paired $t$-test and Wilcoxon signed-rank test were used for comparing both sides at $\mathrm{P}$ value $=0.05$.

Results: The mandible width, IAC width, and IAC height were different between male and female patients. The IMTM roots displayed intimate contact with the IAC in $96.67 \%$ of the cases. The location of the IAC was mostly lingual in both genders. The diameter of the IAC was significant larger in men compared to in the women in terms of width and height.

Conclusions: This study is useful for the dental surgeon to avoid or prevent postoperative numbness or pain, because the awareness of the common location of the IAC and its approximation with the IMTM roots could allow a more cautious and precise approach during IMTM intervention.

Manuscript submitted February 25, 2019, accepted March 18, 2019

aDepartment of Advanced General Dentistry, Faculty of Dentistry, Mahidol University, Bangkok 10400, Thailand

bepartment of Oral and Maxillofacial Surgery, Faculty of Dentistry, Mahidol University, Bangkok 10400, Thailand

${ }^{\mathrm{c}}$ Department of Oral and Maxillofacial Radiology, Faculty of Dentistry, Mahidol University, Bangkok 10400, Thailand

${ }^{\mathrm{d}}$ Walailak University International College of Dentistry $19^{\text {th }}$ floor SM Tower, 979/42-46 Phahonyothin Road, Phaya Thai District, Bangkok 10400, Thailand ${ }^{e}$ Corresponding Author: Natthamet Wongsirichat, Department of Oral and Maxillofacial Surgery, Faculty of Dentistry, Mahidol University, 6 Yothi Street, Rachathewee District, Bangkok 10400, Thailand. Email: natthamet.won@ mahidol.ac.th. Teeranut Chaiyasamut, Department of Oral and Maxillofacial Surgery, Faculty of Dentistry, Mahidol University, 6 Yothi Street, Rachathewee District, Bangkok 10400, Thailand. Email: teeranut.cha@mahidol.ac.th

doi: https://doi.org/10.14740/jocmr3794
Keywords: Cone beam computed tomography; Impacted mandibular third molar; Inferior alveolar canal; Postoperative sequelae; Pain or numbness

\section{Introduction}

The inferior alveolar nerve is a sensory nerve that supplies the mandible area. It initially enters the mandible through the mandibular foramen while some of the inferior alveolar nerve exits at the mental foramen to become the mental nerve where some part of inferior alveolar nerve is the dental plexus nerve for lower anterior teeth. So the inferior alveolar nerve is the most important nerve of the mandible and lower teeth. Iatrogenic injury to the inferior alveolar nerve may cause postoperative pain or numbness after the surgical removal of impacted mandibular third molar (IMTM).

The most common oral surgery is extraction or surgical removal of the third molar [1,2]. The important consideration that is necessary in the mandibular third molar removal procedure is avoidance of inferior alveolar nerve injury. It is well known that the mandibular third molar is usually nearby or in contact with the inferior alveolar canal (IAC). The previous study of Ozturk et al found that the position of the canal was approximately $1 \mathrm{~mm}$ above the inferior border of mandible and passed through mental foramen that was approximately 16.7 $\mathrm{mm}$ above the inferior border of mandible. They studied the skulls of 52 Caucasian patients who were up to 40 years old with cone beam computed tomography (CBCT) [3]. The previous study of Liu et al documented the characteristics of the canal and its relationship with the third molar in 386 panoramic radiographs of Chinese patients who were up to 16 years old, and they concluded that the distal root tip of the third molar was nearest to the canal with a mean distance of approximately $1.27 \pm 1.66 \mathrm{~mm}$ [4]. During the IMTM removal procedure pain may occur even after local anesthesia injection which could indicate the inferior alveolar nerve injury leading to paresthesia, dysesthesia or hypoesthesia of lower lip after the surgery [5].

\section{Panoramic radiograph}

Accordingly, before the surgical removal of IMTM, panoramic radiographs play an important role as a preliminary tool to evaluate the position of the canal and its relationship with the 
Table 1. Age of Patients With the Impacted Mandibular Third Molar in This Study

\begin{tabular}{llll}
\hline \multirow{2}{*}{ Age (years) } & \multicolumn{2}{c}{ Gender } & Total \\
\cline { 2 - 3 } & Male & Female & \\
\hline $20-29$ & 36 & 34 & 70 \\
$30-39$ & 9 & 10 & 19 \\
$40-49$ & 0 & 1 & 1 \\
Total & 45 & 45 & 90 \\
\hline
\end{tabular}

third molar in two dimensions. There are seven classic radiographic signs in the radiograph which show possible risk of inferior alveolar nerve injury: darkening of root, deflection of root, narrowing of root, dark and bifid apex of root, interruption of white line of canal, diversion of canal, and narrowing of canal [6]. Hence, an appropriate radiograph should represent the important adjacent structures of the mandibular third molar which makes the surgery safer by avoiding inferior alveolar nerve injury [7]. However, the panoramic radiograph has wellknown disadvantages such as: distortion of image, magnification and superimposed image with surrounding structures that could erroneously evaluate the distances, position and the relationship between the IMTM and IAC.

\section{CBCT}

CBCT is a digital extraoral radiograph which creates a three-dimensional (3D) image, with no distortion, no superimposed image, and virtually clear image details. Therefore, the CBCT is more advantageous than plain radiography. The CBCT can accurately represent the position of the canal and the relationship between the IMTM and the IAC [8]. It is a medical radiography which was first used in the late 1990s for oral and maxillofacial regions. Nowadays, the $\mathrm{CBCT}$ is widely used for evaluation and determination of the distances and the relationship between the IMTM and the IAC that can show the risk of the inferior alveolar nerve injury to the patients before surgery [9].

\section{Patients and Methods}

\section{Ethic approval}

All of the radiographs in this observational retrospective study were selected from the patients. The protocol of this study was approved by the Committee in the Ethics of Research in Human of the Faculty of Dentistry/Faculty of Pharmacy, Mahidol University, Institutional Review Board (COA.No.MU-DT/PYIRB 2017/049.2509) before data collection.

\section{Patient selection}

The patients were selected based on the following criteria: 1) Selection from the panoramic radiographs with IMTM both
Table 2. The Abbreviation in This Study

Point of reference
1. BB: the outer surface of buccal cortex
2. LB: the outer surface of lingual cortex
3. BC: the buccal border of inferior alveolar canal
4. LC: the lingual border of inferior alveolar canal
5. SC: the superior of inferior alveolar canal
6. IC: the inferior border of inferior alveolar canal
7. AB: the alveolar crest
8. LT: the lower part of tooth
Line of reference
1. BB to LB: mandible width
2. BB to BC: buccal bone width
3. BC to LC: canal width
4. LC to LB: lingual bone width
5. SC to IC: canal height
6. AB to SC: alveolar bone height

sides; 2) From the panoramic radiographs the patients have the CBCT both sides; 3 ) Selection by the date and time, from October 1, 2015 to November 30, 2017; 4) The complete criteria can accept only 90 patients for this study; and 5) The CBCT from the only machine of 3D Accuitomo MCT1 EX2FJ (Morita Mfg. Corp., Kyoto, Japan).

Therefore, these patients took the CBCT at the Oral and Maxillofacial Radiology Clinic, Faculty of Dentistry, Mahidol University by 3D Accuitomo MCT1 EX2FJ (Morita Mfg. Corp., Kyoto, Japan) under standard conditions of manufacturing, set at $60-90 \mathrm{kV}$ and $1-10 \mathrm{~mA}$ with 17.5-s exposure time, $0.125-\mathrm{mm}$ voxel size and $1-\mathrm{mm}$ slice thickness. A personal computer (HP) and i-Dixel One Volume Viewer software program were used for measurement and analyzing data.

Only 180 CBCTs of the IMTM were selected by order of date and time, from October 1, 2015 to November 30, 2017 from 90 Thai patients ( 45 men and 45 women), with both left and right sides of IMTM. The age of patients was 20 years or older (Table 1). The mean age of patients was 27 years old, ranging from 20 to 42 years.

\section{The measurement of this study}

The abbreviations in this study are listed in Table 2.

Figure 1 showed the measurement of nine reference points and seven reference lines. The reference lines are: 1) The distance between the outer surface of buccal cortex and the outer surface of lingual cortex (BB to LB; mandible width); 2) The distance between the outer surface of buccal cortex and the buccal border of IAC (BB to BC; buccal bone width); 3) The distance between the buccal and lingual borders of IAC (BC to LC; canal width); 4) The distance between the lingual border of IAC and the lingual cortex (LC to LB; lingual bone width); 


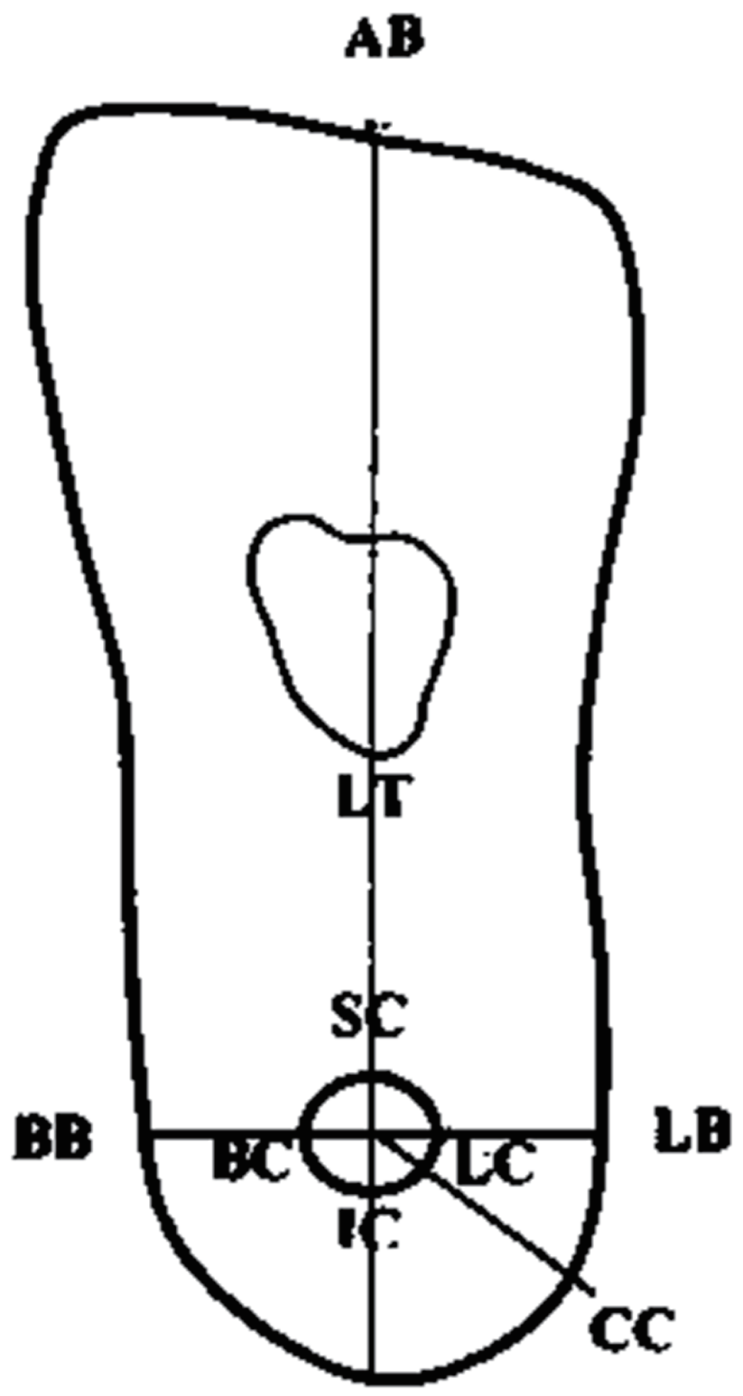

Figure 1. The measurement of nine reference points and seven reference lines of coronal view in cone beam computed tomography.

5) The distance between the superior and inferior borders of IAC (SC to IC; canal height); 6) The distance between the alveolar crest and the superior border of IAC (AB to SC; alveolar bone height); and 7) The distance between the lower part of tooth and the superior border of the IAC (LT to SC).

Before data collection, the intra-examiner was calibrated by repeating the measurement of nine reference points and seven reference lines in 10 patients. Every measurement was made three times with 2-week interval of separating the first and the second measurements. No statistical difference was observed between the measurements ( $\mathrm{P}$ value $>0.05$; paired $t$-test).

\section{Methods}

In the coronal view of the ramus of mandible, the measurements were done in five sections of each tooth: mesial, mid-

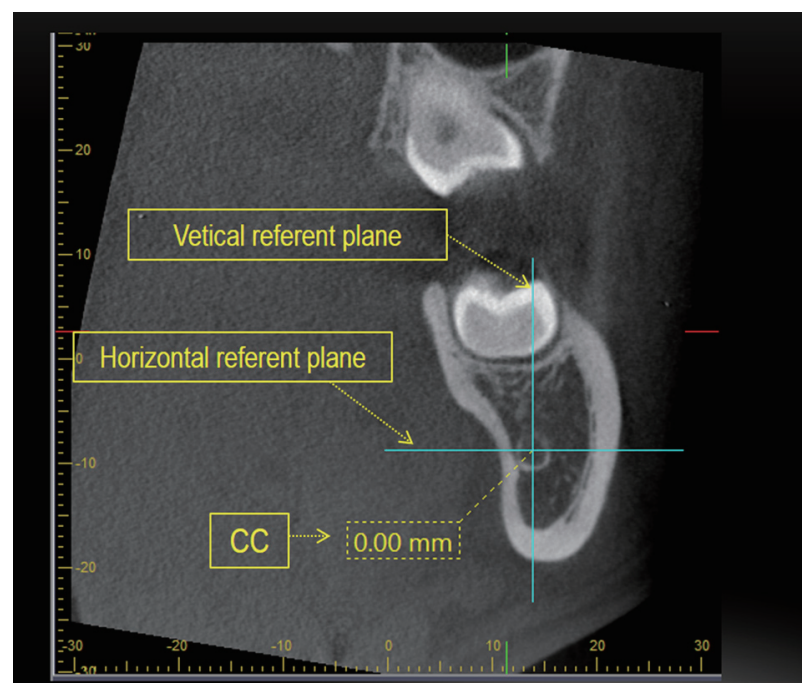

Figure 2. The vertical and horizontal reference planes were traced.

dle, distal, mesial root tip, and distal root tip. Mesial and distal root tips could be the same in some cases that have convergent roots. In this context, an example measurement was done at the mesial section of the coronal view. First, we opened the CBCT data from i-Dixel One Volume Viewer software program and adjusted a sagittal plane for paralleling of first and second molar occlusal planes to the horizontal reference plane and the coronal view automatically appeared in the program. Second, a center point of the IAC (CC) was marked and vertical and horizontal reference planes were traced (Fig. 2). Then, a ruler item in the software program was used for the measurement of the distances at each different point. In this example, the distances of BC to LC (canal width) and SC to IC (canal height) were measured and recorded (Fig. 3).

\section{Data statistical analysis}

In this study, IBM ${ }^{\circledR}$ SPSS $^{\circledR}$ Statistics version 19 was used for analysis. The statistical evaluation included calculation of means and standard deviations. The statistical relation between the result of male and female groups was tested by an independent sample $t$-test and the result of left and right sides group was tested by a paired $t$-test, if the data was normally distributed. But for data which was not normally distributed, the result of male and female groups was tested by the Mann-Whitney U test and the result of the left and right sides group was tested by the Wilcoxon signed-rank test (non-parametric statistics).

\section{Results}

The independent sample $t$-test and Mann-Whitney $\mathrm{U}$ test found statistical differences in the measurement between men and women in the distance of BB to LB (mandible width), BC to LC (canal width), and SC to IC (canal height) (P value $<0.05$ ) (Table 3). No statistical difference was observed ( $\mathrm{P}$ value $>0.05$ ) when comparing left and right IMTM in women but statistical 


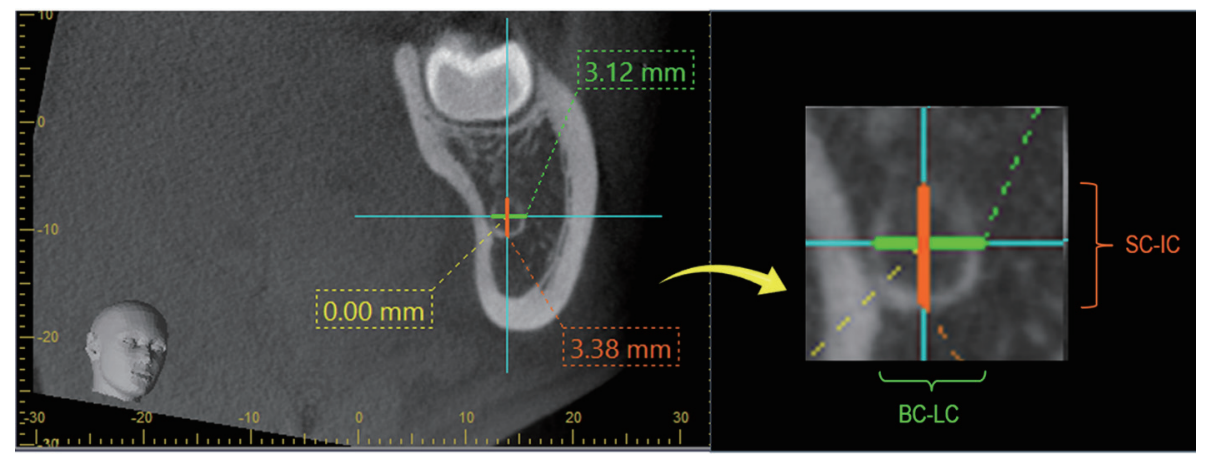

Figure 3. The distances of BC to LC (canal width) and SC to IC (canal height) were measured.

difference was found in men at SC to IC of mesial section (P val$\mathrm{ue}=0.008)$. Measurements of the distances between the IMTM and the IAC at different reference points found that $96.67 \%(87$ from 90 teeth) have contact to the IAC (no distance between the IMTM and the IAC at the different reference points).

\section{The diameter of the IAC}

The diameter of the IAC in men was significantly larger than that in women $(\mathrm{P}$ value $<0.05)$ (Table 3$)$. The average mean of measurements was $2.79 \pm 0.37 \mathrm{~mm}$ at $\mathrm{BC}$ to $\mathrm{LC}$ (canal width) and $3.66 \pm 0.48 \mathrm{~mm}$ at SC to IC (canal height) in women that is vertical oval, and is one fourth of mandibular width. In men, it was $3.35 \pm 0.48 \mathrm{~mm}$ at BC to LC (canal width) (Fig. 4) and 4.31 $\pm 0.62 \mathrm{~mm}$ at SC to IC (canal height) (Fig. 5) that is also vertical oval and is also one fourth of mandibular width (Table 4).

\section{The position of the IAC}

As determined on the $\mathrm{CBCT}$, the IAC was positioned lingual to the IMTM in $76.67 \%$ of cases and $23.33 \%$ were buccal in women. In men, the IAC was found lingual in $71.11 \%$ of cases

Table 3. Comparison of Distances Between Men and Women in Left and Right Teeth

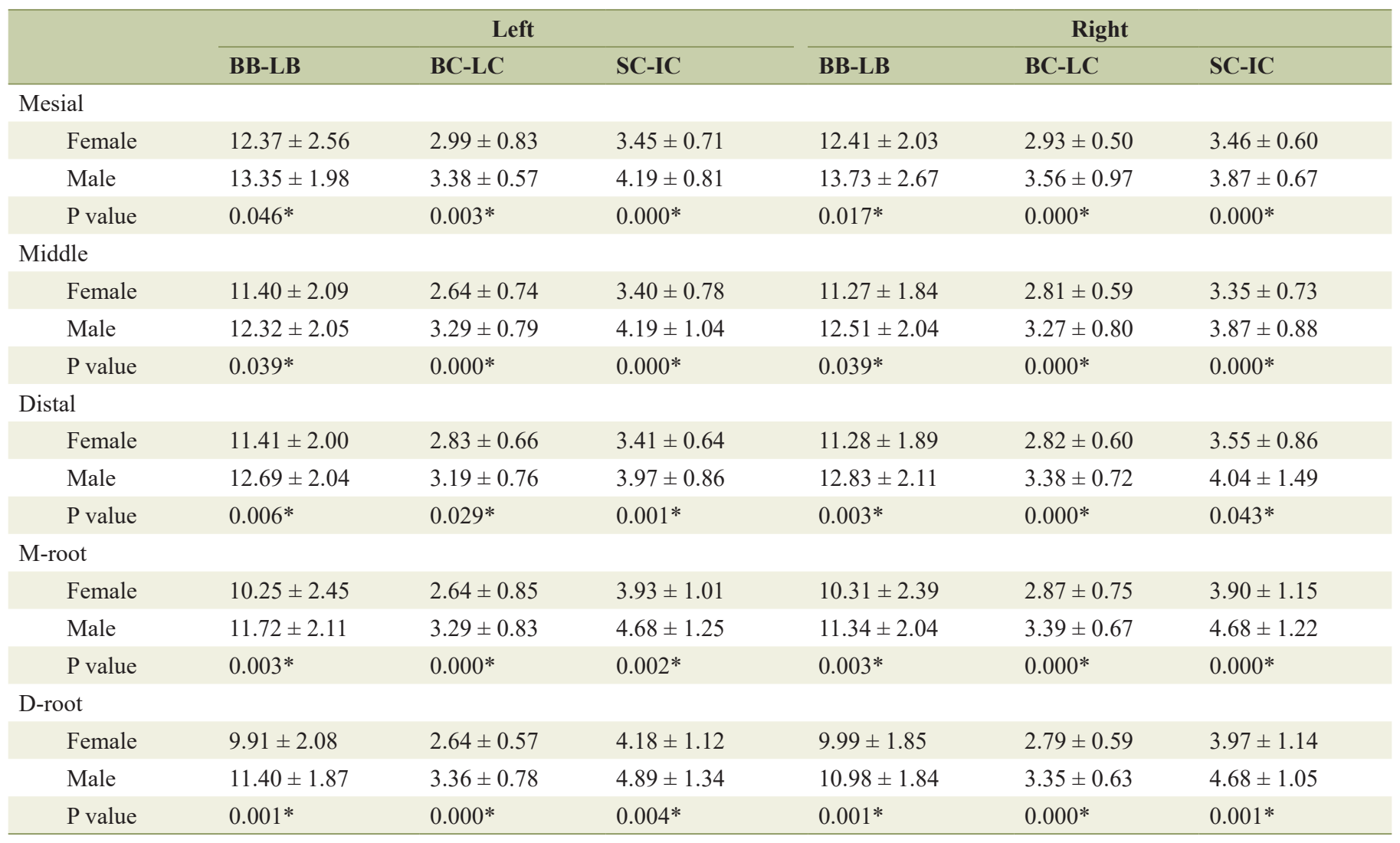

${ }^{*} P$ value $<0.05$ 


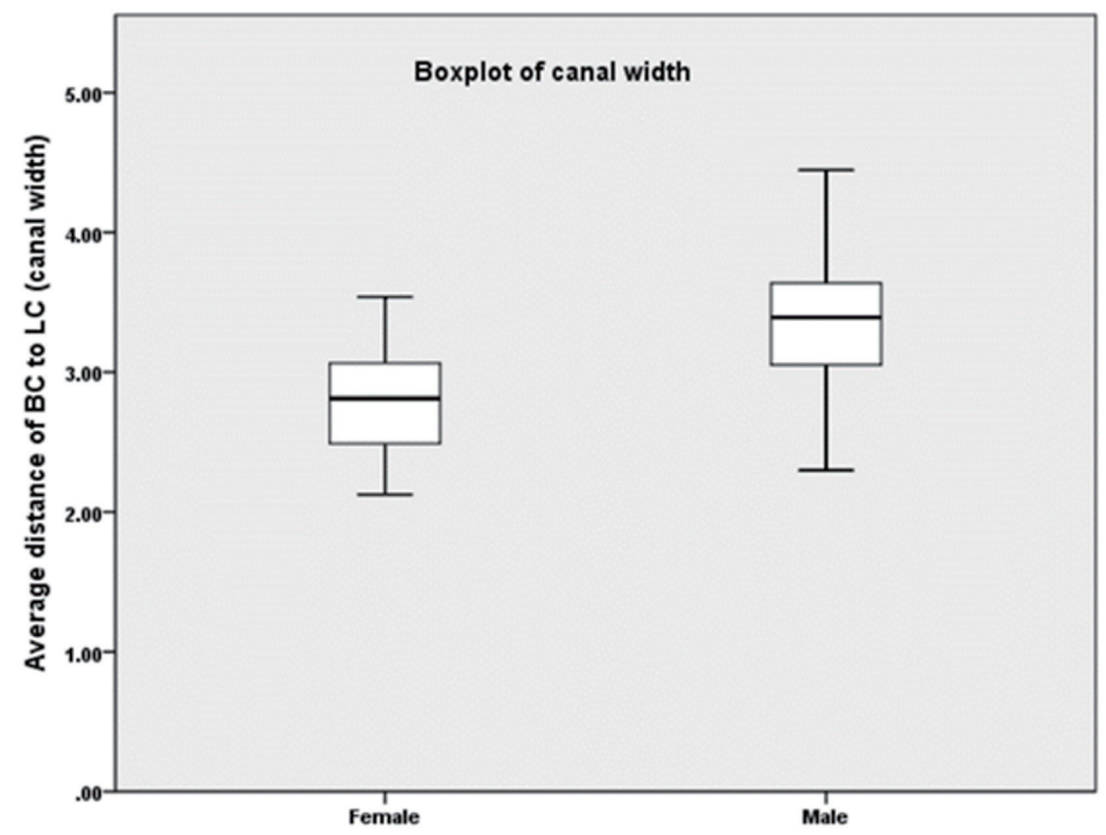

Figure 4. Average distance of BC to LC (Canal width) showed larger canal of men than that of women.

and $28.89 \%$ were buccal. Both were found at lingual $1 / 3$ of mandible width.

\section{Discussion}

This research showed that the IAC was located lingual to the IMTM, which is consistent with the study of Ghaeminia et al
[10] that significantly found 26 from 53 teeth located lingual to the IMTM. In contrast, the study of Miller et al [11], Maegawa et al [12], Yamada et al [13], and Kositbowornchai et al [14] found the IAC located buccally to the IMTM. However, additional investigations, such as the IAC tracing with consecutive scan images, are required to discuss these issues in detail.

The average diameter of the IAC was significantly larger

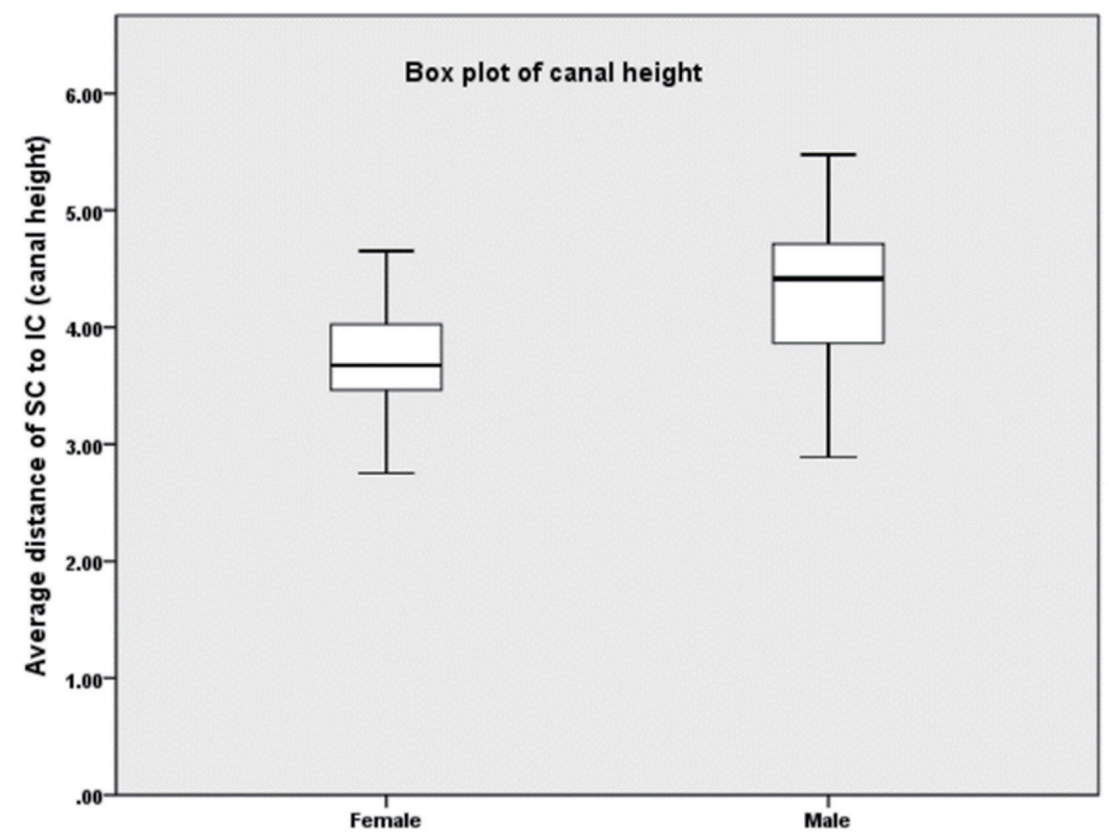

Figure 5. The average distance of SC to IC (canal height) showed larger canal of men than that of women. 
Table 4. The Average Means of Measurements at Each Reference Point

\begin{tabular}{llllllll}
\hline \multirow{2}{*}{ Gender } & \multicolumn{7}{c}{ Average means of measurement } \\
\cline { 2 - 8 } & BB-LB & BB-BC & BC-LC & LC-LB & SC-IC & AB-SC & LT-SC \\
\hline Female & $11.06 \pm 1.77$ & $6.01 \pm 1.77$ & $2.79 \pm 0.37$ & $2.26 \pm 1.27$ & $3.66 \pm 0.48$ & $14.08 \pm 2.14$ & $1.76 \pm 0.96$ \\
Male & $12.29 \pm 1.56$ & $6.39 \pm 1.84$ & $3.35 \pm 0.48$ & $2.55 \pm 1.06$ & $4.31 \pm 0.62$ & $15.52 \pm 2.42$ & $1.69 \pm 1.05$ \\
P value & $0.017^{*}$ & 0.660 & $0.029^{*}$ & 0.997 & $0.002^{*}$ & 0.158 & 0.984 \\
\hline
\end{tabular}

${ }^{*} \mathrm{P}$ value $<0.05$

in male participants than in female participants. This resembles the previous study of Liu et al [4] which was conducted in Chinese participants. The similarity of these results could be attributed to both studies having participants that have similar racial profiles (Mongoloid). Further research in different racial groups could give a broader understanding of the anatomical variations across the world which could help individualized treatment planning and surgical techniques for IMTM intervention.

In this study, the average distance from the IMTM to the superior border of the IAC (LT-SC) was $1.76 \pm 0.96 \mathrm{~mm}$ in women and $1.69 \pm 1.05 \mathrm{~mm}$ in men. The previous study of Momin et al [15] found the mean distance from apex of IMTM roots to canal to be $1.99 \mathrm{~mm}$. The study of Liu et al [4] found that the distance from distal root of the IMTM to the superior border of the IAC was $1.27 \pm 1.66 \mathrm{~mm}$. From the previous studies, it can be concluded that the average distance from tooth to canal is approximately $1-2 \mathrm{~mm}$, and this value can be used for evaluation and prediction before surgical removal of the IMTM.

Nevertheless, the average distance measured on CBCT between the IMTM and the IAC in retrospective studies usually misrepresents the actual average of most IMTM cases. The main reason for this inaccurate representation could be that preliminary panaromic radiographs which do not display classical signs of close relationship between IMTM and IAC were usually not sent for CBCT. In this research, the distances between IMTM and the IAC at different reference points found approximately $97 \%$ contact between both structures.

Therefore, in this study, the statistical difference was in the average measurement of variables according to gender. The comparison between left and right mandibular third molar found no statistical difference in women but statistical difference was observed in men at SC-IC of mesial section only. For the average diameter of the IAC, men showed a significant larger diameter than women.

In conclusion, this study can guide oral surgeons and can be applied to evaluate and predict the relationship between the IMTM and the IAC before surgery. If the IAC was damaged iatrogenically the quality of life of the patient may be disrupted by pain, numbness or altered sensations around the mandible. The oral surgeon can prevent unintended sequelae (numbness and/or intraoperative pain) with CBCT images. We provide useful data for regarding the relationship between the IMTM and the IAC.

\section{Acknowledgments}

The authors would like to thank the staff and dental assistants including colleague and co-workers in the Department of Oral and Maxillofacial Surgery, Faculty of Dentistry, Mahidol University.

\section{Funding}

This research received no any financial support.

\section{Conflict of Interest}

None to declare.

\section{Informed Consent}

Not applicable.

\section{Author Contributions}

None to declare.

\section{References}

1. Laskin DM. Evaluation of the third molar problem. J Am Dent Assoc. 1971;82(4):824-828.

2. Patel S, Mansuri S, Shaikh F, Shah T. Impacted mandibular third molars: a retrospective study of 1198 cases to assess indications for surgical removal, and correlation with age, sex and type of impaction-a single institutional experience. J Maxillofac Oral Surg. 2017;16(1):79-84.

3. Ozturk A, Potluri A, Vieira AR. Position and course of the mandibular canal in skulls. Oral Surg Oral Med Oral Pathol Oral Radiol. 2012;113(4):453-458.

4. Liu T, Xia B, Gu Z. Inferior alveolar canal course: a radiographic study. Clin Oral Implants Res. 2009;20(11):12121218.

5. Hasegawa T, Ri S, Shigeta T, Akashi M, Imai Y, Kakei Y, Shibuya Y, et al. Risk factors associated with inferior alveolar nerve injury after extraction of the mandibular third molar - a comparative study of preoperative images by panoramic radiography and computed tomography. Int J Oral Maxillofac Surg. 2013;42(7):843-851.

6. Rood JP, Shehab BA. The radiological prediction of inferior alveolar nerve injury during third molar surgery. 
Br J Oral Maxillofac Surg. 1990;28(1):20-25.

7. Schulze R, Krummenauer F, Schalldach F, d'Hoedt B. Precision and accuracy of measurements in digital panoramic radiography. Dentomaxillofac Radiol. 2000;29(1):52-56.

8. Scarfe WC, Farman AG, Sukovic P. Clinical applications of cone-beam computed tomography in dental practice. J Can Dent Assoc. 2006;72(1):75-80.

9. De Vos W, Casselman J, Swennen GR. Cone-beam computerized tomography (CBCT) imaging of the oral and maxillofacial region: a systematic review of the literature. Int J Oral Maxillofac Surg. 2009;38(6):609-625.

10. Ghaeminia H, Meijer GJ, Soehardi A, Borstlap WA, Mulder J, Berge SJ. Position of the impacted third molar in relation to the mandibular canal. Diagnostic accuracy of cone beam computed tomography compared with panoramic radiography. Int J Oral Maxillofac Surg. 2009;38(9):964-971.

11. Miller CS, Nummikoski PV, Barnett DA, Langlais RP. Cross-sectional tomography. A diagnostic technique for determining the buccolingual relationship of impacted mandibular third molars and the inferior alveolar neurovascular bundle. Oral Surg Oral Med Oral Pathol.
1990;70(6):791-797.

12. Maegawa H, Sano K, Kitagawa Y, Ogasawara T, Miyauchi K, Sekine J, Inokuchi T. Preoperative assessment of the relationship between the mandibular third molar and the mandibular canal by axial computed tomography with coronal and sagittal reconstruction. Oral Surg Oral Med Oral Pathol Oral Radiol Endod. 2003;96(5):639-646.

13. Yamada T, Ishihama K, Yasuda K, Hasumi-Nakayama Y, Ito K, Yamaoka M, Furusawa K. Inferior alveolar nerve canal and branches detected with dental cone beam computed tomography in lower third molar region. J Oral Maxillofac Surg. 2011;69(5):1278-1282.

14. Kositbowornchai S, Densiri-aksorn W, Piumthanaroj P. Ability of two radiographic methods to identify the closeness between the mandibular third molar root and the inferior alveolar canal: a pilot study. Dentomaxillofac Radiol. 2010;39(2):79-84.

15. Momin MA, Matsumoto K, Ejima K, Asaumi R, Kawai T, Arai Y, Honda K, et al. Correlation of mandibular impacted tooth and bone morphology determined by cone beam computed topography on a premise of third molar operation. Surg Radiol Anat. 2013;35(4):311-318. 